\title{
Prüfgremien müssen konkrete Entscheidungsgründe liefern
}

_ Nach einem Urteil des Landessozialgerichts Nordrhein-Westfalen können Prüfgremien Praxisbesonderheiten in ei-

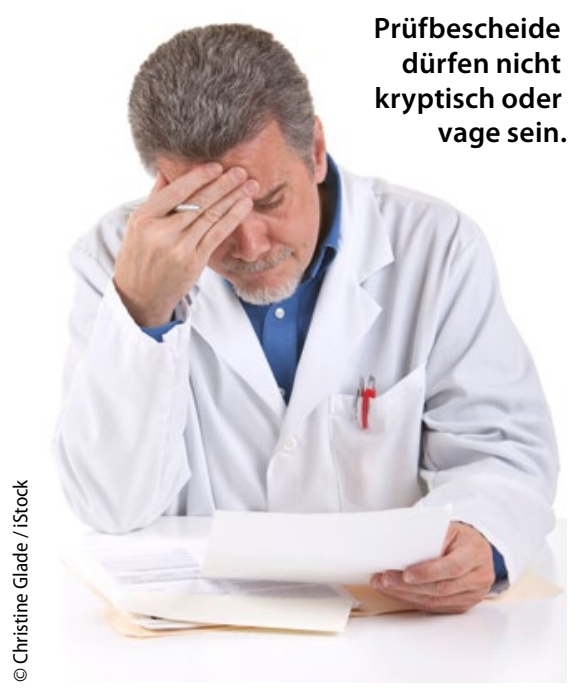

nem Bescheid nicht einfach ohne Begründung ablehnen (Az.: L 11 KA 116/13). Im konkreten Fall ging es um die Rechtmäßigkeit einer schriftlichen Beratung wegen Überschreitung der ArzneimittelRichtgrößen in den vier Quartalen des Jahres 2010, die die Prüfungsstelle im November 2012 anordnete. Die Gemeinschaftspraxis - eine Schwerpunktpraxis in den Disease-Management-Programmen (DMP) Diabetes Typ 1 und 2 - widersprach dem Bescheid mit der Begründung, dass die Praxisbesonderheiten nicht ausreichend berücksichtigt worden wären. Sie beantragte eine individuelle Richtgröße und bot auch eine Einzelfallprüfung ihrer kostenintensiven Patienten an. Die Prüfstelle lehnte dies ab, da sie keine Notwendigkeit gemäß $\$ 5$ Abs. 4 der damaligen regionalen Richtgrößenvereinbarung erkennen konnte.

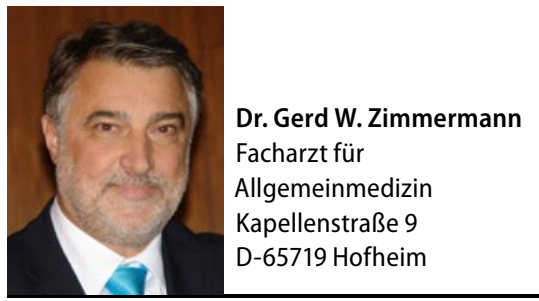

\section{MMW-KOMMENTAR}

Nach Auffassung der Richter genügte diese Mitteilung des Prüfgremiums nicht den Anforderungen an einen nachvollziehbaren $B e-$ scheid, weil er nicht die maßgeblichen Gründe für dessen Ablehnung enthielt. Ein Blick in den § 106 Abs. $5 a$ Satz 1 SGB V, auch in Verbindung mit $A b s$. 5e, verrät uns, dass eine Beratung nur dann in Betracht kommt, wenn die Überschreitung des Richtgrößenvolumens um mehr als 25\% nicht durch Praxisbesonderheiten begründet ist. Solche Besonderheiten, so das Gericht, gehen aus einer Zusammensetzung der Patienten hervor, die in den Praxen der Vergleichsgruppe nicht anzutreffen ist. Für ein Gericht sei der Begriff nur einschränkt überprüfbar. Das Prüfgremium müsse deshalb konkret darlegen, wie das Behandlungsverhalten des Arztes bewertet wurde und auf welchen Erwägungen die betroffene Kürzungsmaßnahme beruht.

\section{Hausarzt ist Lotse bei ambulanten Operationen}

_ Ambulante Operationen sind fast ausschließlich im fachärztlichen Bereich angesiedelt. Hausärzte könnten zwar durchaus Leistungen aus dem zugrunde liegenden Katalog nach $\$ 115$ b SGB V erbringen. Da die Abrechnung allerdings nicht nur an die fachliche Qualifikation, sondern auch an eine sehr kostenaufwändige Raumausstattung gebunden ist, scheitert dies an der betriebswirtschaftlichen Machbarkeit. Genau umgekehrt ist die Ausgangssituation allerdings bei der OP-Vorbereitung und ggf. auch der OP-Nachsorge. Hier sieht der EBM Exklusivleistungen für den hausärztlichen Bereich vor, ohne die ein Operateur erst gar nicht tätig werden kann. Besonders wichtig ist dabei, dass diese Leistungen ohne eine besondere Anforderung an die Praxisausstattungen und extrabudgetär vergütet werden.

\section{MMW-KOMMENTAR}

Die präoperativen Leistungen nach den Nrn. 31010-31013 EBM dürfen nur von Fachärzten für Allgemeinmedizin bzw. Innere und Allgemeinmedizin, praktischen Ärzten, Ärzten ohne Gebietsbezeichnung, an der hausärztlichen Versorgung teilnehmenden Fachärzten für Innere Medizin sowie von Fachärzten für Kinder- und Jugendmedizin berechnet wer- den. Man muss beachten, dass diese Leistungen nur vor ambulanten Operationen nach Kapitel 31.2 und belegärztlichen Operationen nach Kapitel 36.2 bzw. ambulanten Operationen nach § $115 b$ SGB V berechnungsfähig sind. Ausgeschlossen sind die sogenannten kleinen chirurgischen Eingriffe, z. B. nach den Nrn. 02300-02302. Hier gibt es eine bemerkenswerte Ausnahme: Wird ein solcher Eingriff unter Narkose bei Neugeborenen, Säuglingen, Kleinkindern oder Kindern bis zum vollendeten 12. Lebensjahr durchgeführt, kommt dafür die Nr. 31101 zum Ansatz. In diesem Fall kann für die OP-Vorbereitung die Nr. 31010 abgerechnet werden. 